\title{
DETERMINAÇÃO ESPECTROFOTOMÉTRICA DE DIPIRONA EM PRODUTOS FARMACÊUTICOS POR INJEÇÃO EM FLUXO PELA GERAÇÃO DE ÍONS TRIIODETO
}

Airton Vicente Pereira*, Luciane Penckowski, Marcos Vosgerau e Michelli Fernanda Sassá

Departamento de Ciências Farmacêuticas, Setor de Ciências Biológicas e da Saúde, Universidade Estadual de Ponta Grossa, CP 195, 84030-900 Ponta Grossa - PR

Orlando Fatibello Filho

Departamento de Química, Centro de Ciências Exatas e de Tecnologia, Universidade Federal de São Carlos, CP 676, 13565-975 São Carlos - SP

Recebido em 9/5/01; aceito em 7/11/01

\begin{abstract}
SPECTROPHOTOMETRIC DETERMINATION OF METAMIZOL IN PHARMACEUTICAL FORMULATIONS BY FLOW INJECTION TRIIODIDE GENERATION. A flow injection spectrophotometric procedure was developed for the determination of metamizol in pharmaceutical formulations. The system is based on the reaction between metamizol and triiodide generated in the system by mixing iodate and iodide-starch solutions. The absorbance of triiodide-starch complex giving a steady-state baseline value which was monitored at $580 \mathrm{~nm}$. The inverse peaks caused by metamizol samples were measured and there was a direct relationship between absorbance decreasing and metamizol concentration from $1.4 \times 10^{-4}$ to $7.0 \times 10^{-4} \mathrm{~mol} \mathrm{~L}^{-1}$. The RSD was $0.45 \%$ for a metamizol solution $4.2 \times 10^{-4} \mathrm{~mol} \mathrm{~L}^{-1}(\mathrm{n}=10)$ with a detection limit (three-fold blank standard deviation/slope) of $6.0 \times 10^{-5} \mathrm{~mol} \mathrm{~L}^{-1}$ The feasibility of the system was demonstrated for the determination of metamizol in commercial samples with sixty results obtained per hour. The results obtained for metamizol in pharmaceutical formulations using the proposed flow procedure and those obtained using an iodimetric procedure are in agreement at the $95 \%$ confidence level and within an acceptable range of error.
\end{abstract}

Keywords: metamizol; triiodide generation; flow injection spectrophotometry; pharmaceutical formulations.

\section{INRODUÇÃO}

A dipirona (ácido 1-fenil-2,3-dimetil-5-pirazolona-4metilaminometanossulfônico) (Figura 1) é o analgésico antipirético mais utilizado no Brasil. Quimicamente, a dipirona é um derivado 5pirazolônico com a presença de um grupo metanossulfônico na estrutura ${ }^{1}$. É comercializada principalmente na forma sódica em diferentes formulações farmacêuticas (solução oral, injetável, comprimidos e supositórios).

A Farmacopéia Brasileira ${ }^{2}$ preconiza a iodimetria para a determinação quantitativa da dipirona sódica (matéria-prima). O método consiste em solubilizar a amostra com água deionizada, acidificá-la com $\mathrm{HCl} 0,02 \mathrm{~mol} \mathrm{~L}^{-1}$ e titular com uma solução padronizada de iodo $0,05 \mathrm{~mol} \mathrm{~L}^{-1}$, utilizando-se como indicador uma solução de amido $1 \% \mathrm{~m} / \mathrm{v}$ adicionada próximo ao ponto final. A titulação iodimétrica da dipirona está baseada na oxidação, em meio ácido, do grupo metanossulfônico a sulfato, de acordo com a reação:

$$
\mathrm{RHSO}_{3}^{-}+\mathrm{I}_{3}^{-}+\mathrm{H}_{2} \mathrm{O} \rightleftharpoons \mathrm{RSO}_{4}^{2-}+3 \mathrm{I}^{-}+3 \mathrm{H}^{+}
$$

O reagente azul de tetrazólio foi utilizado na determinação colorimétrica da dipirona em produtos farmacêuticos ${ }^{3}$. A reação da dipirona com ácido sulfúrico produz formaldeído que reage com o ácido 5-amino-a-naftolssulfônico formando um produto de cor amarela que se torna azul pela diluição com água. Esta reação já foi aplicada na determinação colorimétrica ${ }^{4}$ da dipirona em comprimidos, sangue e urina na faixa de $10-40 \mu \mathrm{g} \mathrm{mL}^{-1}$. Estas reações são lentas e requerem aquecimento limitando o número de amostras a serem analisadas.

*e-mail: airtonvp@uepg.br

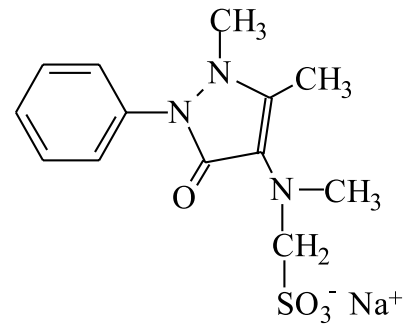

Figura 1. Fórmula estrutural da dipirona sódica

A espectrofotometria ultravioleta $(280 \mathrm{~nm})$ permitiu a determinação de dipirona como único princípio ativo em algumas amostras, mas esse procedimento é inadequado para dipirona em associação com outros fármacos, uma vez que estes absorvem nessa região do espectro eletromagnético. Entretanto, a espectrofotometria ultravioleta-derivativa ${ }^{5}$ permitiu a determinação da mistura ternária contendo dipirona, paracetamol e cafeína presentes em comprimidos.

A cromatografia em camada delgada ${ }^{6}$ foi utilizada para a determinação dos principais metabólitos da dipirona no soro e na urina. Após a separação cromatográfica, procedeu-se a detecção espectrofotométrica em $265 \mathrm{~nm}$. A cromatografia líquida de alta eficiência (CLAE) tem sido aplicada principalmente quando há a necessidade da separação da dipirona em associação com outros fármacos ${ }^{7}$ e de seus metabólitos em amostras complexas como o plasma humano ${ }^{8}$.

A análise por injeção em fluxo permite minimizar erros decorrentes da manipulação de soluções, diminuir custos e aumentar a freqüência de análise sobretudo quando um grande número de amostras deve ser analisado. Diversos sistemas por injeção em fluxo têm sido empregados no controle de qualidade de medicamentos ${ }^{9}$. Siste- 
mas por injeção em fluxo com detecção amperométrica ${ }^{10} \mathrm{e}$ espectrofotométrica ${ }^{11}$ foram aplicados na determinação da dipirona em produtos farmacêuticos.

O emprego da análise por injeção em fluxo permite a geração em linha de íons $\mathrm{I}_{3}^{-}$a partir de soluções de iodato e iodeto de potássio ${ }^{12}$, em um meio de acidez controlada, conforme a reação ${ }^{13}$ :

$$
\mathrm{IO}_{3}^{-}+8 \mathrm{I}^{-}+6 \mathrm{H}^{+} \rightleftharpoons 3 \mathrm{I}_{3}^{-}+3 \mathrm{H}_{2} \mathrm{O}
$$

Neste trabalho, foi desenvolvido um sistema por injeção em fluxo para a geração de íons $\mathrm{I}_{3}^{-}$e esse foi empregado na determinação de dipirona em produtos farmacêuticos. O sistema desenvolvido dispensa a preparação e padronização de soluções de iodo e supera o problema da instabilidade desta solução pela exposição ao ar, uma vez que as reações ocorrem em um sistema fechado e em poucos segundos. Os íons triiodeto gerados em linha, ao reagirem com a dipirona injetada no sistema, oxidam o grupo metanossulfônico do fármaco a sulfato. $\mathrm{O}$ consumo de íons $\mathrm{I}_{3}^{-}$pela dipirona provoca um decréscimo da absorbância (linha base) do complexo triiodeto-amido que é monitorado espectrofotometricamente em $580 \mathrm{~nm}$.

\section{PARTE EXPERIMENTAL}

\section{Equipamentos}

No sistema por injeção em fluxo desenvolvido neste trabalho, foi utilizada uma bomba peristáltica Ismatec de 8 canais, modelo 761850 com tubos de propulsão de Tygon ${ }^{\circledR}$ de diferentes diâmetros internos; tubos de polietileno de $0,8 \mathrm{~mm}$ de diâmetro interno e conectores de acrílico. As soluções de referência ou de amostras foram injetadas utilizando-se um injetor-comutador duplo manual construído em acrílico e as medidas de absorbância foram obtidas utilizando-se um espectrofotômetro Micronal, modelo B 342 equipado com uma célula espectrofotométrica com caminho óptico de $1,0 \mathrm{~cm}$.

\section{Reagentes e soluções}

A solução estoque de dipirona $2,8 \times 10^{-3} \mathrm{~mol} \mathrm{~L}^{-1}$ foi preparada diariamente, dissolvendo-se $50 \mathrm{mg}$ de dipirona sódica em balão volumétrico de $50 \mathrm{~mL}$ com água deionizada. Essa solução foi utilizada para preparar as soluções de referência por diluições apropriadas.

A solução de iodeto de potássio $2,5 \times 10^{-3} \mathrm{~mol} \mathrm{~L}^{-1}$ - amido a 0,1 $\% \mathrm{~m} / \mathrm{v}$ foi preparada transferindo-se $10 \mathrm{~g}$ de amido solúvel para um béquer de $1 \mathrm{~L}$ e adicionados cerca de $800 \mathrm{~mL}$ de água deionizada. A solução foi aquecida até a completa solubilização do amido. Após o resfriamento, foram adicionados $0,42 \mathrm{~g}$ de iodeto de potássio (Synth). A solução foi transferida para um balão volumétrico de $1 \mathrm{~L}$ e o volume foi completado com água deionizada.

A solução estoque de iodato de potássio $1,0 \times 10^{-3} \mathrm{~mol} \mathrm{~L}^{-1}$ foi preparada com 0,2142 $\mathrm{g}$ de iodato de potássio (Merck) dissolvidos em água deionizada e o volume completado para $1 \mathrm{~L}$ em balão volumétrico. Esta solução foi utilizada nas diluições subseqüentes.

\section{Preparo das amostras}

Amostras contendo dipirona nas formulações farmacêuticas solução, injetável e comprimidos foram adquiridas em farmácias da cidade de Ponta Grossa e analisadas pelo método proposto.

\section{a) Comprimidos}

Foram pesados e macerados 10 comprimidos até pó fino. Uma massa desse pó correspondente a $500 \mathrm{mg}$ de dipirona foi transferida para um balão volumétrico de $500 \mathrm{~mL}$ e adicionou-se água deionizada até completar esse volume. Após agitação, a solução foi filtrada através de papel de filtro, $15 \mathrm{~mL}$ do filtrado foram transferidos para um balão volumétrico de $100 \mathrm{~mL}$ e o volume completado com água deionizada. Alíquotas de $50 \mu \mathrm{L}$ dessas soluções das amostras foram injetadas utilizando-se um injetor manual.

\section{b) Injetável e Solução oral (gotas)}

Foram transferidos volumes correspondentes a $500 \mathrm{mg}$ de dipirona para balões volumétricos de $500 \mathrm{~mL}$ e adicionou-se água deionizada até completar esse volume. Alíquotas de $15 \mathrm{~mL}$ de cada uma dessas soluções foram transferidas para balões volumétricos de $100 \mathrm{~mL}$ e o volume completado com água deionizada. Alíquotas de $50 \mu \mathrm{L}$ das soluções das amostras foram injetadas utilizando-se um injetor manual.

\section{Sistema de injeção em fluxo}

O sistema por injeção em fluxo proposto baseia-se na geração em linha de íons triiodeto. As soluções de iodato de potássio em meio ácido e iodeto de potássio contendo amido misturam-se a partir do ponto de confluência $\mathrm{x}_{1}$. A geração de íons triiodeto ocorre na bobina helicoidal $\mathrm{B}_{1} \mathrm{e}$, imediatamente, devido a presença do amido, ocorre a formação do complexo triiodeto-amido de cor azul. Quando a alavanca do injetor manual é movimentada no sentido indicado (Figura 2), a solução de dipirona é inserida no percurso analítico e arrastada pela solução transportadora. A reação da dipirona com 0 complexo triiodeto-amido ocorre na bobina helicoidal $\mathrm{B}_{2}$, ocasionando um decréscimo no sinal da absorbância (diminuição da intensidade da cor azul devido ao consumo de triiodeto) continuamente monitorada em $580 \mathrm{~nm}$. Esse decréscimo de absorbância foi proporcional à concentração de dipirona injetada no sistema em fluxo.

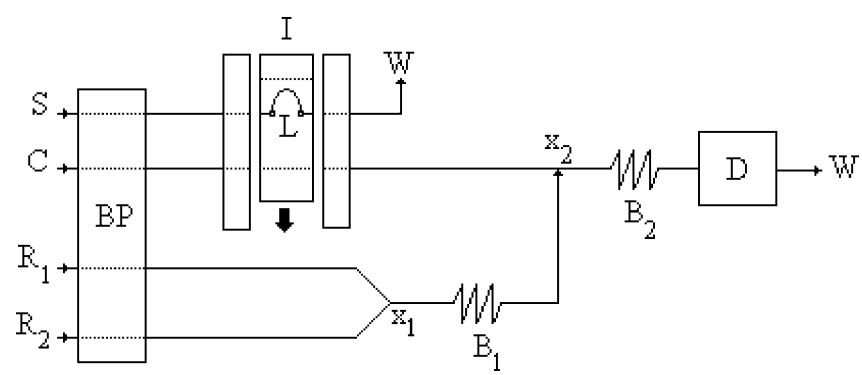

Figura 2. Diagrama de fluxo proposto para a determinação de dipirona. $B P$, bomba peristáltica; $C$, solução transportadora $\left(\mathrm{HCl} 1,0 \times 10^{-2} \mathrm{~mol} \mathrm{~L}^{-1}\right)$ com uma vazão de $2,7 \mathrm{~mL} \mathrm{~min}^{-1} ; R_{l}$, solução de $\mathrm{KIO}_{3} 6 \times 10^{-5} \mathrm{~mol} \mathrm{~L}^{-1} \mathrm{em} \mathrm{HCl}$ $1 \times 10^{-2} \mathrm{~mol} \mathrm{~L} L^{-1} \mathrm{com}$ uma vazão de $1,6 \mathrm{~mL} \mathrm{~min}^{-1} ; R_{2}$, solução de KI 2,5 $x$

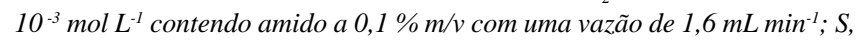
soluções de amostras; I, injetor na posição de aspiração da amostra; $L$, alça de amostragem (10 cm; $50 \mu L) ; B_{1}$ e $B_{2}$, bobinas de reação de 300 e $325 \mathrm{~cm}$, respectivamente; $x_{1}$ e $x_{2}$, pontos de confluência; $D$, espectrofotômetro $(580 \mathrm{~nm}) \mathrm{eW}$, descarte

\section{RESULTADOS E DISCUSSÃO}

\section{Geração de íons triiodeto}

O sistema em fluxo proposto foi baseado na reação entre o iodato de potássio e iodeto de potássio, em meio ácido, para produzir íons triiodeto. Inicialmente, o sistema foi montado para averiguar a efici- 
ência na geração de íons triiodeto. O sistema em fluxo apresentado na Figura 2, permitiu a geração em linha dos íons triiodeto seguida da imediata complexação com o amido presente na solução de iodeto de potássio. A cor azul do complexo iodo-amido foi monitorada em $580 \mathrm{~nm}$. O sistema em fluxo permitiu a obtenção de valores estáveis de absorbância (linha de base) evidenciando, assim, a geração contínua e reprodutível de íons triiodeto. Da mesma forma, a introdução de uma alíquota de $20 \mu \mathrm{L}$ de solução de dipirona sódica $1,0 \mathrm{mg} \mathrm{mL}$ ${ }^{1}$ permitiu constatar a reação da dipirona com o complexo amidoiodo, devido a diminuição do sinal analítico (absorbância).

\section{Estudo dos parâmetros do sistema em fluxo}

Os parâmetros do sistema de injeção em fluxo foram otimizados para obtenção dos melhores resultados em termos de sensibilidade e freqüência analítica.

O efeito da concentração de iodato de potássio sobre a geração de íons triiodeto foi avaliado nas concentrações de $1,0 \times 10^{-5}$ a $6,0 \times 10^{-5}$ mol L-1 (soluções em $\mathrm{HCl} 1,0 \times 10^{-3} \mathrm{~mol} \mathrm{~L}^{-1}$ ) utilizando-se como $\mathrm{R}_{2}$ uma solução de iodeto de potássio $0,1 \mathrm{~mol} \mathrm{~L}^{-1}$ contendo amido a $0,1 \%$ $\mathrm{m} / \mathrm{v}$ e água deionizada como transportador. Conforme apresentado na Figura 3, os valores da linha base aumentaram até atingir o valor de 1 unidade de absorbância com a solução de iodato de potássio $6,0 \mathrm{x}$ $10^{-5} \mathrm{~mol} \mathrm{~L}^{-1}$. Portanto, a solução do reagente iodato de potássio $6,0 \times 10^{-5} \mathrm{~mol} \mathrm{~L}^{-1}$ foi escolhida para os experimentos posteriores.

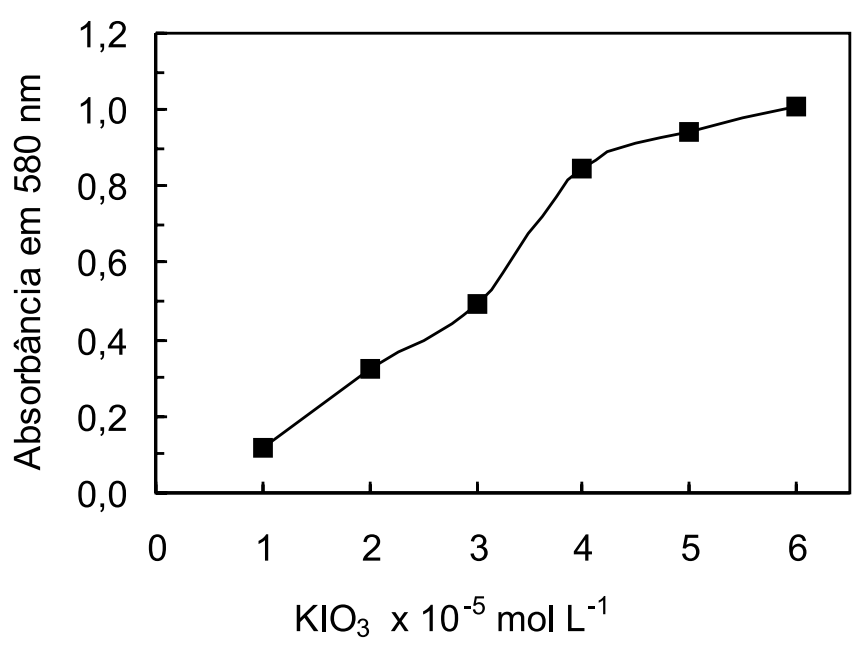

Figura 3. Efeito da concentração do iodato de potássio sobre o sinal de absorbância da linha base

O efeito da concentração de iodeto de potássio na geração de triiodeto foi avaliado na faixa de concentração de $1,0 \times 10^{-3}$ a $1,0 \times$ $10^{-2} \mathrm{~mol} \mathrm{~L}{ }^{-1}$ mantendo-se a concentração de amido $0,1 \% \mathrm{~m} / \mathrm{v}$. O gráfico apresentado na Figura 4 mostra que os sinais de absorbância aumentaram com o aumento da concentração de iodeto de potássio até uma concentração de $5,0 \times 10^{-3} \mathrm{~mol} \mathrm{~L}^{-1}$, acima da qual permaneceu constante. Portanto, a solução do reagente iodeto de potássio 2,5 x $10^{-3} \mathrm{~mol} \mathrm{~L}^{-1}$ foi escolhida para os experimentos posteriores.

O efeito da concentração do ácido clorídrico utilizado na preparação das soluções de iodato de potássio sobre a geração dos íons triiodeto foi avaliado na faixa de concentração de 5,0 x $10^{-3}$ a 3,0 x $10^{-2} \mathrm{~mol} \mathrm{~L}{ }^{-1}$. Os sinais de absorbância da linha base aumentaram com o aumento da concentração de ácido até uma concentração de $1,0 \times 10^{-2} \mathrm{~mol} \mathrm{~L}^{-1}$, conforme apresentado na Figura 5. Portanto, a solução de ácido clorídrico de $1,0 \times 10^{-2} \mathrm{~mol} \mathrm{~L}^{-1}$ foi escolhida para a preparação das soluções de iodato de potássio $6,0 \times 10^{-5} \mathrm{~mol} \mathrm{~L}^{-1}$.

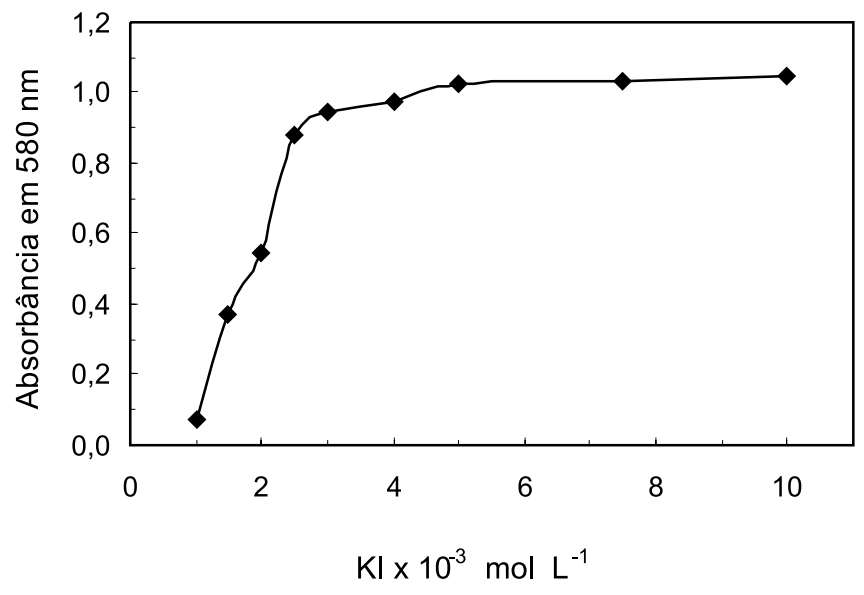

Figura 4. O efeito da concentração de iodeto de potássio sobre o sinal de absorbância da linha base

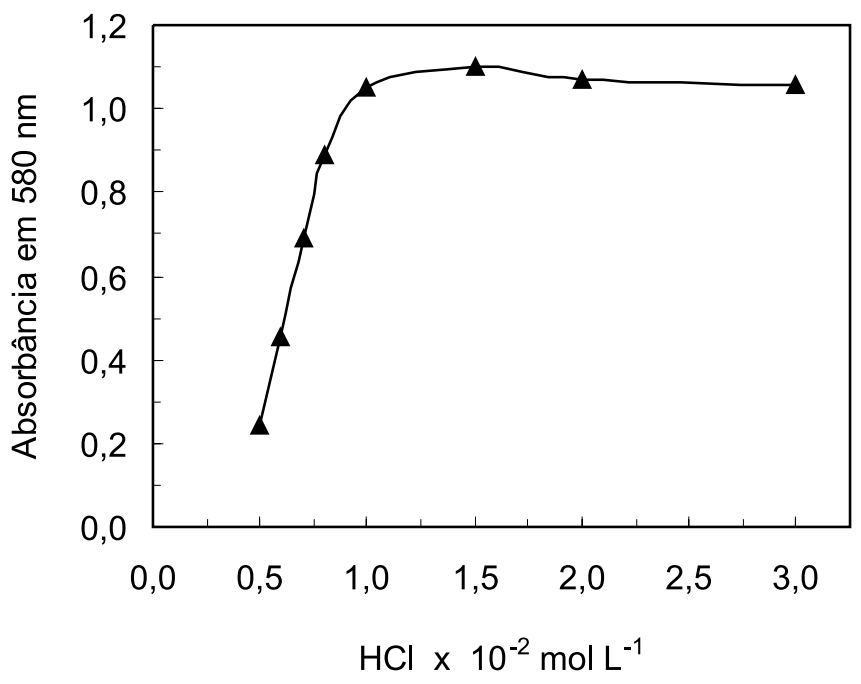

Figura 5. Efeito da concentração do ácido clorídrico utilizado nas soluções de iodato de potássio sobre a geração dos íons triiodeto

A concentração de amido presente na solução de iodeto de potássio foi estudada na faixa de 0,025 a $0,15 \% \mathrm{~m} / \mathrm{v}$. Verificou-se um aumento dos valores de absorbância até a concentração máxima avaliada. Como a solução com concentração de amido de $0,15 \% \mathrm{~m} / \mathrm{v}$ apresentou uma ligeira turbidez decorrente da limitada solubilidade do amido, a concentração de $0,1 \% \mathrm{~m} / \mathrm{v}$ foi selecionada para o restante do trabalho.

Nos experimentos anteriores, a água deionizada foi utilizada como transportador. Estudou-se o efeito da utilização de solução de $\mathrm{HCl}$ como transportador no intervalo de concentração de $1,0 \times 10^{-3}$ a 2,0 x $10^{-2} \mathrm{~mol} \mathrm{~L}^{-1}$ sobre a linha base. Ocorreu um ligeiro aumento da linha base até a máxima concentração de $\mathrm{HCl}$ avaliada. A solução de $\mathrm{HCl} 1,0 \times 10^{-2} \mathrm{~mol} \mathrm{~L}^{-1}$ foi então utilizada como solução transportadora, uma vez que foi constatada pequena diferença entre as linhas base obtidas com as soluções $1,0 \times 10^{-2} \mathrm{~mol} \mathrm{~L}^{-1}$ e $2,0 \times 10^{-2} \mathrm{~mol} \mathrm{~L}^{-1}$.

A influência do comprimento dos reatores helicoidais $\mathrm{B}_{1}(100,150$, $250,300$ e $400 \mathrm{~cm})$ e $B_{2}(125,225,325$ e $400 \mathrm{~cm})$ sobre a geração de íons triiodeto foi estudada fixando-se inicialmente um reator $\mathrm{B}_{2}$ de $225 \mathrm{~cm}$. Os valores de absorbância da linha base aumentaram e com o aumento do comprimento desses reatores até $400 \mathrm{~cm}$. Reatores helicoidais de 300 e $325 \mathrm{~cm}$, respectivamente foram escolhidos como adequados em termos de sensibilidade e estabilidade da linha base. 
O volume da amostra inserida no sistema foi avaliado utilizando-se comprimentos da alça de amostragem (L) de 10, 15, 20, 25 e $30 \mathrm{~cm}$. Para uma solução de dipirona com concentração de $2,8 \mathrm{x}$ $10^{-4} \mathrm{~mol} \mathrm{~L}^{-1}$, a magnitude do sinal analítico aumentou até $30 \mathrm{~cm}$ $(150 \mu \mathrm{L})$. Entretanto, uma alça de amostragem de $10 \mathrm{~cm}(50 \mu \mathrm{L})$ foi escolhido para obtenção de uma frequiência analítica mais elevada, mantendo-se a sensibilidade do procedimento para a determinação de dipirona na faixa de $10^{-4} \mathrm{~mol} \mathrm{~L}^{-1}$.

O efeito da vazão da solução transportadora foi avaliada nos valores 1,0, 2,0 2,7 e 4,0 mL min ${ }^{-1}$, mantendo-se constantes as vazões das soluções de iodato de potássio e iodeto de potássio-amido. Utilizando-se soluções de dipirona com concentrações entre $1,4 \times 10^{-4}-$ $5,6 \times 10^{-5} \mathrm{~mol} \mathrm{~L}^{-1}$, a sensibilidade do procedimento em fluxo diminuiu com o aumento da vazão, provavelmente em conseqüência da diminuição do tempo de reação. Uma vazão de $2,7 \mathrm{~mL} \mathrm{~min}^{-1}$ foi escolhida para a solução transportadora.

As vazões dos reagentes $R_{1}$ e $R_{2}$ foram variadas simultaneamente nos valores de $0,9,1,6,2,4$ e 3,0 $\mathrm{mL} \mathrm{min}^{-1}$, mantendo-se a vazão

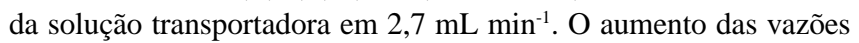
da solução reagente resultou na diminuição dos valores de absorbância. Nesse caso, vazões de 1,6 $\mathrm{mL} \mathrm{min}^{-1}$ foram selecionadas para os reagentes $R_{1}$ e $R_{2}$ como uma combinação adequada entre sensibilidade e frequiência analítica.

\section{Estudo de interferentes em potencial e teste recuperação}

O efeito de interferentes em potencial na determinação de dipirona em formulações farmacêuticas foi avaliado para excipientes normalmente presentes em amostras comerciais. Nesses experimentos, foram utilizadas soluções de referência de dipirona $2,8 \times 10^{-4} \mathrm{~mol} \mathrm{~L}^{-1}$ adicionadas com cada um dos possíveis interferentes (carboximetilcelulose, cloreto de sódio, sacarina, glicose, sacarose, estearato de magnésio, lactose e cafeína) em concentrações de $2,8 \times 10^{-3} \mathrm{~mol} \mathrm{~L}^{-1}$ e os resultados foram comparados com aqueles obtidos com uma solução de referência de dipirona $2,8 \times 10^{-4} \mathrm{~mol} \mathrm{~L}^{-1}$. Nenhuma das substâncias testadas causou qualquer interferência na determinação de dipirona.

Nos testes de adição e recuperação do analito, três concentrações diferentes de dipirona foram adicionadas a quatro amostras de formulações farmacêuticas e os resultados comparados com aqueles obtidos com as amostras não adicionadas do analito. Os resultados estão apresentados na Tabela 1 . Os valores percentuais de recuperação variaram entre 98,8 e 101,0 \% sugerindo assim ausência de interferência da matriz no procedimento em fluxo.

Tabela 1. Resultados do teste de recuperação da dipirona adicionada às formulações farmacêuticas empregando o sistema em fluxo

\begin{tabular}{lccc}
\hline & \multicolumn{2}{c}{ Dipirona $\left(\mathrm{mg} \mathrm{mL}^{-1}\right)$} & Recuperação \\
\cline { 2 - 3 } Amostra & Adicionado & Recuperado* & $(\%)$ \\
\hline Anador & 50 & 49,7 & 99,4 \\
& 100 & 100,3 & 100,3 \\
Conmel & 150 & 150,1 & 100,1 \\
& 50 & 50,4 & 100,8 \\
& 100 & 99,9 & 99,9 \\
Dexalgen & 150 & 149,3 & 99,5 \\
& 50 & 50,5 & 101,0 \\
& 100 & 99,7 & 99,7 \\
Magnopyrol & 150 & 149,7 & 99,8 \\
& 50 & 49,4 & 98,8 \\
& 100 & 100,2 & 100,2 \\
& 150 & 150,6 & 100,4 \\
\hline
\end{tabular}

$\mathrm{n}=5$

\section{Curva analítica e aplicações}

O sistema em fluxo apresentou uma curva analítica no intervalo de concentração de $1,4 \times 10^{-4}$ a $7,0 \times 10^{-4} \mathrm{~mol} \mathrm{~L}^{-1}$ de dipirona $(\Delta \mathrm{A}=$ $0,0217+0,0832 \mathrm{C} ; \mathrm{r}=0,9998)$ onde $\Delta \mathrm{A}$ é a variação de absorbância e $\mathrm{C}$ a concentração molar de dipirona). A curva analítica está apresentada na Figura 6. O método proposto apresentou um limite de detecção de $6,0 \times 10^{-5} \mathrm{~mol} \mathrm{~L}^{-1}$ (três vezes o desvio padrão do branco/ inclinação da curva analítica), um desvio padrão relativo de $0,45 \%$ ( $\mathrm{n}=10$ ) para uma concentração de dipirona de $4,2 \times 10^{-4} \mathrm{~mol} \mathrm{~L}^{-1} \mathrm{e}$ uma freqüência analítica de 60 determinações por hora. Um fiagrama da curva analítica está apresentado na Figura 7.

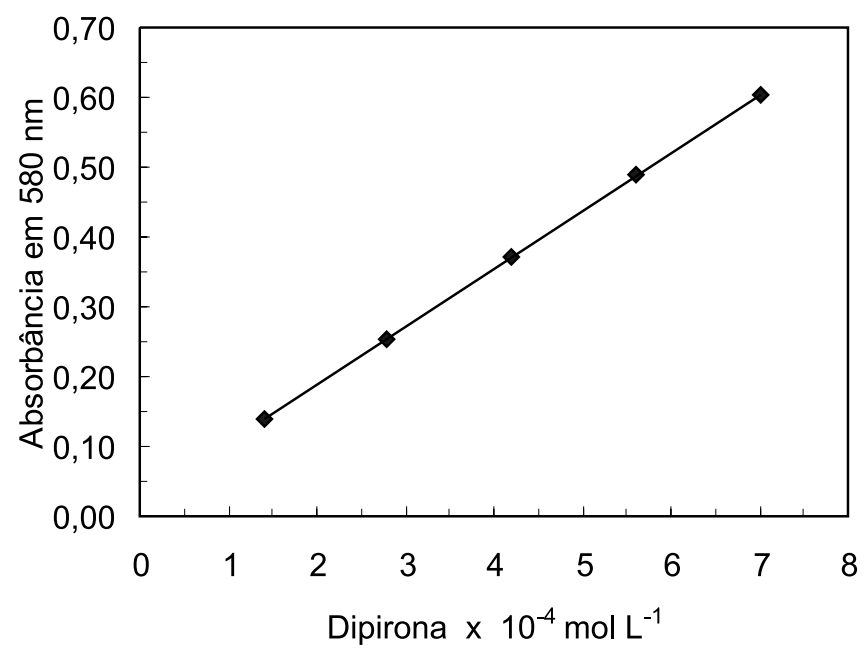

Figura 6. Curva analítica da determinação de dipirona sódica

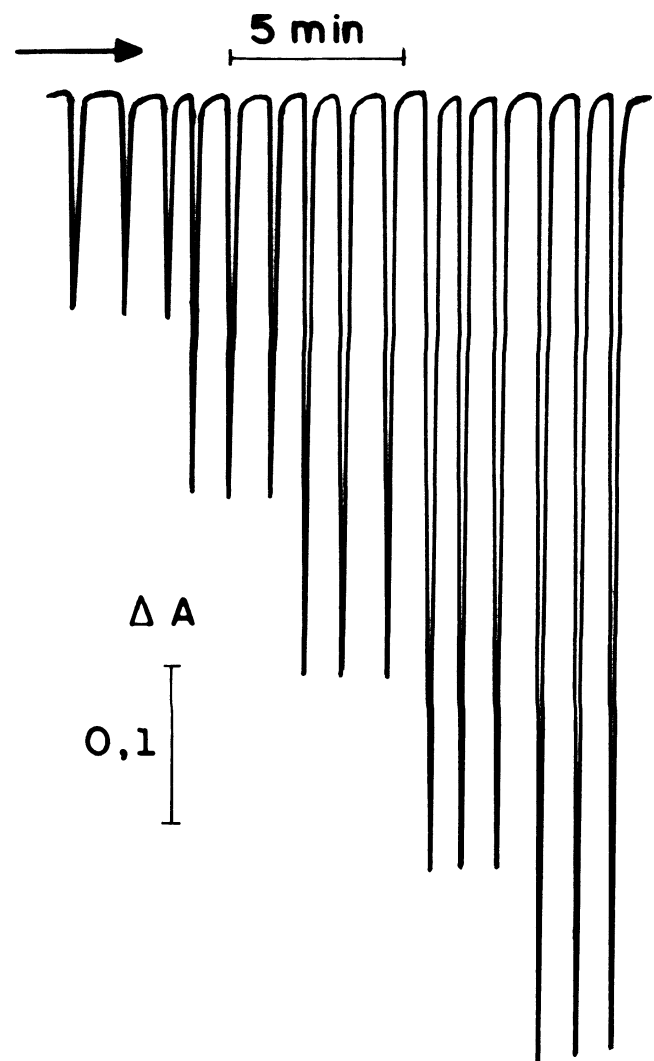

Figura 7. Fiagrama típico da curva analítica obtido com o sistema em fluxo proposto para a determinação de dipirona . Da esquerda para a direita: triplicata das soluções de referência de dipirona nas concentrações de 1,4, 2,8, 4,2, 5,6 e 7,0 x $10^{-4} \mathrm{~mol} \mathrm{~L}^{-1}$ 
O procedimento por injeção em fluxo foi aplicado na determinação de dipirona em diversos produtos farmacêuticos (Tabela 2). Aplicando-se o test $t$ pareado para os teores de dipirona encontrados empregando-se o procedimento em fluxo proposto e aqueles teores encontrados empregando um procedimento iodimétrico ${ }^{2}$, encontrouse que os resultados são concordantes a um nível de $95 \%$ de confiança. Esse mesmo resultado foi encontrado no teste $\mathrm{t}$ pareado com os valores rotulados.

Tabela 2. Determinação de dipirona em produtos farmacêuticos pelo sistema por injeção em fluxo e por iodimetria

\begin{tabular}{lccccc}
\hline Amostras & Rotulado & Iodimetria & FIA $^{2}$ & $\mathrm{Er}_{1}(\%)$ & $\mathrm{Er}_{2}(\%)$ \\
\hline Anador $^{\circledR}$ & $50{ }^{*}$ & $505 \pm 2$ & $501 \pm 1$ & $-0,8$ & $+0,2$ \\
Anador $^{\circledR}$ & $500^{+}$ & $498 \pm 2$ & $505 \pm 1$ & $+1,4$ & $+1,0$ \\
Conmel $^{\circledR}$ & $320^{+}$ & $327 \pm 3$ & $317 \pm 1$ & $-3,1$ & $-0,9$ \\
Dexalgen $^{\circledR}$ & $500^{*}$ & $502 \pm 2$ & $504 \pm 1$ & $+0,4$ & $+0,8$ \\
Novalgina $^{\circledR}$ & $500^{*}$ & $486 \pm 3$ & $488 \pm 1$ & $+0,4$ & $-2,4$ \\
Novalgina $^{\circledR}$ & $500^{+}$ & $517 \pm 3$ & $508 \pm 1$ & $-1,7$ & $+1,6$ \\
Magnopyrol $^{\circledR}$ & $500^{*}$ & $512 \pm 2$ & $497 \pm 1$ & $-2,9$ & $-0,6$ \\
Magnopyrol $^{\circledR}$ & $500^{+}$ & $520 \pm 2$ & $515 \pm 1$ & $-1,0$ & $+3,0$ \\
\hline
\end{tabular}

* valores em $\mathrm{mg} \mathrm{mL}^{-1+}$ valores em mg comprimido ${ }^{-1}$

$\mathrm{Er}_{1}$ FIA vs iodimetria $\mathrm{Er}_{2}$ FIA vs rotulado

Portanto, o sistema por injeção em fluxo desenvolvido permitiu a geração de íons triiodeto com obtenção de uma linha base estável e a determinação quantitativa da dipirona em produtos farmacêuticos com rapidez, simplicidade e economia de reagentes como vantagens quando comparado com a iodimetria.

\section{AGRADECIMENTOS}

Os autores agradecem ao Programa PIBIC/CNPq/UEPG pelas bolsas de Iniciação Científica concedidas a L. Penckowski e M. F. Sassá.

\section{REFERÊNCIAS}

1. Korolkovas, A,; Burckhalter, J. H.; Química Farmacêutica, Guanabara Koogan: Rio de Janeiro, 1988, p.193.

2. Farmacopéia Brasileira, 3ํe ed., Atheneu Ed.: São Paulo, 1977.

3. Vassileva, A. P.; Mikrochim. Acta 1976, 3-4, 247.

4. Diab, A. H.; Pharmazie 1977, 32, 226.

5. Dinc, E.; Onur, F.; Anal. Chim. Acta 1998, 359, 93.

6. Sistovaris, N.; Pola, W.; Wolhoff, H.; J. Chromatogr. 1983, 274, 289.

7. Abounassif, M. A.; Gad-Kariem, E. A.; Wahbi, A. M.; Farmaco 1990, 45, 465.

8. Carretero, I.; Vadillo, J. M.; Laserna, J. J.; Analyst 1995, 120, 1729.

9. Martínez-Calatayud, J.; Flow Injection Analysis of Pharmaceuticals, Taylor \& Francis: Hants, 1996, p.120.

10. Pérez Ruiz, T.; Martínez, L. C.; Tomás, V.. J.; Pharm. Biomed. Anal. 1994, $12,1109$.

11. Bautista, J. A. G.; Zamora, L. L.; Mateo, J. V. G.; Martínez-Calatayud, J. M.; Anal. Lett. 1996, 29, 2667.

12. Hernández-Méndez, J.; Mateos, A. A.; Parra, A.; Maria, G. C.; Anal. Chim. Acta 1986, 184, 243.

13. Harris, D. C.; Quantitative Chemical Analysis, $3^{\text {rd }}$ ed., W. H. Freeman and Company: New York, 1991, p. 411 